\title{
Sensibilidade de plantas de café micorrizadas à herbicidas ${ }^{1}$
}

\author{
Sensibility of coffee plants micorrized to herbicides
}

Felipe Paolinelli Carvalho ${ }^{2}$; André Cabral França ${ }^{3}$; Miguel Henrique Rosa Franco ${ }^{4}$; Moisés Avelar $^{5}$; Samuel Dias Moreira ${ }^{4}$; Ademilson Oliveira Alecrim ${ }^{4}$ José Barbosa dos Santos ${ }^{6}$

Resumo - Objetivou-se com esse trabalho avaliar o efeito de herbicidas aplicados em pósemergência em plantas de café arábica inoculadas com fungos micorrízicos arbusculares. $\mathrm{O}$ experimento foi conduzido em esquema fatorial 2 × 6, sendo mudas de café arábica cultivar Catuaí IAC 99 inoculadas e não inoculadas com fungos micorrízicos arbusculares (Fator 1), e cinco herbicidas (chlorimuron-ethyl, oxyfluorfen, fluazifop-p-butil, fomesafen, [fluazifop-pbutil+fomesafen]) e uma testemunha (Fator 2), com quatro repetições. As mudas inoculadas com fungos micorrízicos arbusculares (Rhizophagus clarus e Gigaspora margarita) e produzidas em sacolas plásticas contendo solo e esterco (3:1) foram transplantadas para vasos de $20 \mathrm{dm}^{3}$ de solo, no estádio de cinco pares de folhas. No estádio de sete pares de folhas foram submetidas a aplicação dos herbicidas. Após a aplicação do herbicida foram mensuradas: porcentagem de intoxicação, altura, área foliar, volume radicular, matéria seca das folhas e raízes, teor nutricional da folha e colonização radicular. Observou-se que a aplicação de chlorimuron-ethyl, oxyfluorfen e [fluazifopp-butil+fomesafen] resultaram em menor incremento das variáveis e maior intoxicação. $\mathrm{O}$ fluazifop-p-butil apresentou maior seletividade para as plantas de café. As plantas inoculadas obtiveram maior colonização radicular de fungos micorrízicos que as não inoculadas. Apenas o fluazifop-p-butil não causou interferência na colonização, sendo que as plantas inoculadas mostraram menor intoxicação que as não inoculadas. Considerando todos os parâmetros avaliados conclui-se que as plantas de café arábica cultivar Catuaí Vermelho IAC 99 inoculadas com fungos micorrízicos arbusculares apresentaram maior tolerância ao fluazifop-p-butil. Da mesma forma, conclui-se também que plantas de café quando inoculadas com FMAs apresentaram-se mais tolerantes aos efeitos negativos dos herbicidas chlorimuron-ethyl, fomesafen, oxyfluorfen e [fluazifop-p-butil+fomesafen].

Palavras-chaves: Coffea arabica, fungos micorrízicos arbusculares, seletividade, tolerância

\footnotetext{
Abstract - The objective of this work was to evaluate the effects of herbicides applied in postemergence of Arabic coffee plants inoculated and non-inoculated with arbuscular mycorrhizal fungi. The experiment was conducted in a factorial arrangement 2 x 6, being seedlings of Arabic coffee (Catuaí IAC $99 \mathrm{cv}$ ) inoculated and non-inoculated with mycorrhizal fungi (Factor 1), and five herbicides (chlorimuron-ethyl, oxyfluorfen, fluazifop-p-butil, fomesafen, [fluazifop-p-butil + fomesafen]) and a control treatment (Factor 2), with four replications. The seedlings inoculated

${ }^{1}$ Recebido para publicação em 10/10/2013 e aceito em 05/07/2015.

2 Pós-doutorando (PNPD/Capes) - Universidade Federal de Viçosa. Florestal, Minas Gerais, Brasil. felipepaolinelli@yahoo.com.br (*autor para correspondência)

${ }^{3}$ Professor Adjunto - Universidade Federal dos Vales do Jequitinhonha e Mucuri. Diamantina, Minas Gerais, Brasil

${ }^{4}$ Mestre em Produção Vegetal - UFVJM. Diamantina, Minas Gerais, Brasil

${ }^{5}$ Engenheiro agrônomo - UFVJM. Diamantina, Minas Gerais, Brasil

${ }^{6}$ Professor Adjunto - UFVJM. Diamantina, Minas Gerais, Brasil
} 
with mycorrhizal fungi (Rhizophagus clarus and Gigaspora margarita) were produced in polyethylene bags and transplanted to $20 \mathrm{dm}^{3}$ pots when it was in five pairs leaves stage. In seven pairs leaves stage the plants were submitted to the herbicide applications. After 45 days were measured: percentage of intoxication, height, leaf area, root volume, leaves and roots dry matter, leaf nutrient content and root colonization. Noted that chlorimuron-ethyl, oxyfluorfen and [fluazifop-p-butil+fomesafen] resulting in less increment of variables and higher phytotoxicity. The fluazifop-p-butil showed greater selectivity to coffee plants. The inoculated plants obtained higher root colonization than non-inoculated. Considering all parameters, it is concluded that the Arabica coffee cultivar Catuaí Vermelho IAC 99 inoculated with mycorrhizal fungi showed higher tolerance to fluazifop-p-butil. Similarly, also concludes that coffee plants inoculated with FMAs had become more tolerant to the adverse effects of chlorimuron-ethyl, fomesafen, oxyfluorfen and [fluazifop-p-butil + fomesafen].

Keywords: Coffea arabica, arbuscular mycorrhizal fungi, selectivity, tolerance

\section{Introdução}

O cafeeiro é uma cultura perene e pode produzir por mais de 30 anos. Apesar de ter características instáveis, como os ciclos de preço e fisiológicos de produção (bienalidade), a cafeicultura é atividade economicamente interessante, pois é de alta densidade financeira e rentável em pequena escala. Além disso, a cafeicultura gera maior renda e emprego por unidade de área em relação a outras culturas, sendo adequada à topografia montanhosa por permitir diversidade de processos de produção (Caixeta et al., 2008).

A produção da cafeicultura mantém um crescimento constante, demonstrando que a maior utilização da mecanização, aliada às inovações tecnológicas, às exigências do mercado, à qualidade do produto e à boa gestão da atividade, são fatores extremamente importantes e necessários para o seu avanço e modernização (CONAB, 2013). No entanto, falhas relacionadas ao controle de plantas daninhas na cultura do café podem estar dentre os principais fatores que contribuem para a queda na produção das lavouras brasileiras.

Plantas jovens de café são muito sensíveis à interferência das plantas daninhas que ocorrem na linha de plantio, podendo ter seu crescimento e ciclo reprodutivo comprometidos caso o controle não seja efetuado em tempo hábil (Ronchi et al., 2001). Em razão da competição com as plantas daninhas, sua produtividade e qualidade podem ser severamente comprometidas. Por isso, o manejo de plantas daninhas na lavoura constitui-se uma das principais práticas que oneram o custo de produção (Silva e Ronchi, 2003). Além disso, devido à rusticidade das plantas daninhas, estas se destacam na rapidez e eficiência da utilização dos recursos do ambiente, levando vantagem sobre o crescimento das culturas. Segundo Ronchi et al. (2007), geralmente as plantas daninhas apresentam maior concentração de nutrientes nos tecidos, devido à maior habilidade ou eficiência na absorção do que as plantas de café.

Manejar plantas daninhas na linha de plantio do café é extremamente complicado, pois o controle manual é dispendioso e, muitas vezes, impossível de ser realizado, haja vista a escassez de mão-de-obra e a umidade excessiva do solo. Nesse sentido, o controle químico carece de herbicidas seletivos aplicados em pré ou pós-emergência das plantas daninhas para uso nessa fase da cultura e, também, de tecnologias adequadas à aplicação de herbicidas não-seletivos (Ronchi et al., 2001).

As micorrizas arbusculares são associações simbiotróficas entre fungos da classe dos glomeromicetos e raízes da maioria das plantas vasculares (Colozzi Filho e Cardoso, 2000). Os fungos micorrizícos arbusculares (FMA) são compostos por filamentos (hifas) que penetram nas raízes, onde passam a 
funcionar como sistema radicular adicional, aumentando a área radicular efetivamente ocupada no solo, melhorando a capacidade das plantas em absorverem nutrientes, e beneficiando seu crescimento, desenvolvimento e, consequentemente, seu vigor e sua produtividade (Moreira e Siqueira, 2006).

A produção de mudas de cafeeiro pode ser favorecida pela obtenção de mudas de alta qualidade e homogeneidade em curtos períodos de tempo (Locatelli e Lovato 2002). Desta forma, a inoculação com espécies de FMA, favorece o crescimento das mudas (PouyuRojas e Siqueira, 2000), aumenta a tolerância ao estresse provocado pelo transplantio, aumenta o índice de sobrevivência após o transplantio, além de reduzir o tempo para formação da muda no viveiro (Miranda et al., 2006). O alto potencial do uso de fungos micorrízicos na formação de mudas de cafeeiro é caracterizado pela elevada dependência micorrízica do cafeeiro, principalmente, em solos de baixa fertilidade (Colozzi Filho et al., 1994).

Portanto, o objetivo deste trabalho foi avaliar a seletividade de herbicidas aplicados em pós-emergência em plantas de café submetidas ou não a inoculação com fungos micorrizícos arbusculares.

\section{Material e Métodos}

$\mathrm{O}$ experimento foi realizado em casa de vegetação, na área experimental do Departamento de Agronomia da Universidade Federal dos Vales do Jequitinhonha e Mucuri. Utilizaram-se sementes de Catuaí Vermelho IAC-99.

As sementes, depois de lavadas com solução de hipoclorito de sódio (1\%), foram colocadas para germinar em areia autoclavada e, quando se apresentavam na fase de "palito de fósforo", foram repicadas para sacolas plásticas com substrato adubado, de acordo com Guimarães et al. (1999). No ato da repicagem, metade das mudas foram inoculadas, aplicandose $10 \mathrm{~g}$ de inoculante por plântula. $\mathrm{O}$ inoculante foi composto pela mistura de espécies de fungos micorrizicos arbusculares (FMAs) Rhizophagus clarus e Gigaspora margarita (1:1), que apresentava aproximadamente 100 esporos por $10 \mathrm{~g}$ do inoculante.

Com quatro a cinco pares de folhas definitivas, as mudas foram transplantadas para vasos de $20 \mathrm{~L}$ preenchidos com substrato composto por solo peneirado e esterco de curral curtido (3:1). Para fornecimento de $\mathrm{P}_{2} \mathrm{O}_{5}$, utilizou-se superfosfato simples (100 g por vaso), além de calcário dolomítico, a fim de elevar a saturação por bases a $60 \%$. Os cálculos foram desenvolvidos de acordo com os resultados descritos na análise. Aos 30 dias após o plantio, efetuou-se a adubação de cobertura, de acordo com a Comissão de Fertilidade do Solo de Minas Gerais (Guimarães et al., 1999).

O delineamento experimental adotado foi o de blocos casualizados, com esquema fatorial $2 \times 6$, com quatro repetições. $O$ primeiro fator correspondeu em avaliar o efeito da associação micorrízica, planta inoculada e não inoculada, e o segundo fator referente à aplicação de cinco herbicidas em pósemergência (chlorimuron-ethyl a $15 \mathrm{~g}$ i.a ha ${ }^{-1}$; fluazifop-p-butil $200 \mathrm{~g}$ i.a ha ${ }^{-1}$; fomesafen $250 \mathrm{~g}$ i.a. ha ${ }^{-1}$ [fluazifop-p-butil+fomesafen] 250+200 g i.a ha ${ }^{-1}$; oxyfluorfen $600 \mathrm{~g}$ i.a ha ${ }^{-1}$ ) e uma testemunha sem a aplicação. As unidades experimentais foram estabelecidas como sendo um vaso, preenchido com $20 \mathrm{dm}^{3}$ de solo, com uma muda de cafeeiro.

A aplicação dos herbicidas foi efetuada no estádio de sete pares de folhas desenvolvidos, com auxílio de um pulverizador costal pressurizado a $\mathrm{CO}_{2}$, mantido a pressão constante de $250 \mathrm{kPa}$, munido com uma barra com duas pontas tipo leque (TT 110.02), espaçadas a $50 \mathrm{~cm}$ entre si e proporcionando volume de calda de $200 \mathrm{~L} \mathrm{ha}^{-1}$.

Aos 15 dias após a aplicação dos herbicidas (DAA), avaliou-se, visualmente, a porcentagem de fitointoxicação das plantas em relação ao controle sem herbicida, atribuindo-se notas que variaram entre 0 e $100 \%$, em que $0 \%$ 
correspondeu à ausência de sintomas visíveis e $100 \%$ à morte das plantas.

Aos zero e 60 dias após a aplicação dos herbicidas, foram mensuradas a altura, o diâmetro do caule, a área foliar, a densidade radicular e a matéria seca do caule, folhas e raiz das mudas de café. Após determinação da altura e diâmetro do caule, as plantas foram divididas em folha, caule e raiz. O método do scanner foi utilizado para mensuração da área foliar, sendo posteriormente calculados pelo programa DAA (Determinador Digital de Áreas). A densidade radicular foi determinada pelo método do volume deslocado em proveta graduada (Carrigan e Frey, 1980). Posteriormente, as partes das plantas foram colocadas em estufa de circulação forçada de ar $\left(60^{\circ} \mathrm{C}\right)$, para determinação da massa seca.

Após a secagem das amostras promoveu-se moagem por planta em moinhos de lâminas do tipo Willey, equipado com peneira fina (40 mesh), visando maior homogeneização do material. Amostras desse material vegetal moído foram submetidas à digestão nitroperclórica. Em seguida, foram determinadas as concentrações de $\mathrm{P}$, pelo método da vitamina C modificado (Braga e Defelipo, 1974); de K por fotometria de chama; de $\mathrm{S}$ por turbidimetria do sulfato (Jackson, 1958); e Ca, Mg, Fe, $\mathrm{Zn}, \mathrm{Mn} \mathrm{e} \mathrm{Cu}$ por espectrofotometria de absorção atômica (AOAC, 1975). A digestão sulfúrica do material vegetal foi realizada, para determinação da concentração de $\mathrm{N}$ (nitrogênio total) pelo método de Kjeldahl (1883).

Também foi determinado a porcentagem de colonização aos 60 DAA antes da secagem do material, procedendo com a coleta de amostras do sistema radicular da cultura. Essas amostras foram lavadas, retirando-se fragmentos de aproximadamente $1 \mathrm{~cm}$ de comprimento das raízes finas. A colonização micorrízica radicular foi estimada pelo método da contagem em placa marcada, após a descoloração e posterior coloração, conforme sugerido por Giovanetti e Mosse (1980).
Os dados foram submetidos à análise de variância, com a média das variáveis sendo comparadas pelo teste de Tukey, a 5\% de probabilidade.

\section{Resultados e Discussão}

Houve diferença significativa $(\mathrm{p}<0,05)$ entre os herbicidas aplicados sobre as plantas de café, e entre as plantas inoculadas e não inoculadas com fungos micorrízicos arbusculares (FMAs), para todas as variáveis analisadas.

As plantas tratadas com chlorimuronethyl, oxyfluorfen e [fluazifop-pbutil+fomesafen] apresentaram fitointoxicação, ao contrário do fluazifop-p-butil que não apresentou sintomas de danos visuais. As plantas inoculadas se diferiram das não inoculadas com FMAs, quando tratadas com chlorimuron-ethyl, oxyfluorfen e [fluazifop-pbutil+fomesafen], constatando menor sintomas de danos nas plantas inoculadas (Tabela 1).

Resultados semelhantes foram encontrados por outros autores como Ronchi e Silva (2003), que relataram os principais sintomas de danos visuais do oxyfluorfen em mudas de café em casa de vegetação foram necroses espalhadas pela lâmina foliar, caracterizada por $62,5 \%$ de fitointoxicação. Neste trabalho também se contatou fitointoxicação de 29,5\% do chlorimuron-ethyl, assim como encarquilhamento das folhas para o fluazifop-p-butil+fomesafen. Os mesmos autores Ronchi e Silva (2004), relataram resultados semelhantes, com os mesmos produtos, em plantas de café submetidas aplicação no campo. Magalhães et al. (2012) em aplicação de oxyfluorfen em plantas de café no campo observaram fitointoxicação de 29,4\% após 30 dias da aplicação. Já Alcântara et al. (2000) avaliando herbicidas na fase inicial de crescimento do cafeeiro obtiveram resultados semelhantes quanto a aplicação de fluazifop-pbutil.

Os sintomas de danos descritos para o chlorimuron-ethyl, oxyfluorfen e [fluazifop-p- 
butil + fomesafen] estão estreitamente relacionados com seus respectivos mecanismos de ação (Silva et al., 2009). A mistura [fluazifop-butil+fomesafen], apesar de quando aplicada isoladamente não ter apresentado efeito negativo para as plantas de café, quando utilizados conjuntamente na forma de mistura pronta, esses herbicidas, que agem inibindo a acetil coezima-A caboxilase (fluazifop-p-butil) e inibido ação da enzima PROTOX (fomesafen), causou sintomas de fitointoxicação.

Para o efeito dos herbicidas na altura de plantas de café inoculadas, constatou-se que chlorimuron-ethyl e oxyfluorfen promoveram os menores valores desta variável. Com relação as plantas não inoculadas observou-se que, além do chlorimuron-ethyl e oxyfluorfen, a mistura [fluazifop-p-butil+fomosafen] também promoveu redução na altura das plantas. Quanto a inoculação, apenas o chlorimuron-ethyl e [fluazifop-p-butil+fomesafen] diferiram entre inoculadas e não inoculadas, onde a planta inoculada foi superior às plantas não inoculadas (Tabela 1).

Ao estimar o efeito dos herbicidas sobre a área foliar das plantas de café evidenciou-se que chlorimuron-ethyl, [fluazifop-pbutil+fomosafen] e oxyfluorfen promoveram menor área foliar para ambos os tratamentos. Quanto ao efeito de interação entre os inoculantes e herbicidas observou-se que presença de todos os herbicidas reduziu a área foliar das plantas não inoculadas, enquanto que na testemunha não houve diferença.

Tabela 1. Fitointoxicação aos 15 DAA, altura e área foliar aos 60 DAA em plantas de café (Catuaí Vermelho IAC 99) inoculadas e não inoculadas com fungos micorrízicos arbusculares (Rhizophagus clarus e Gigaspora margarita).

\begin{tabular}{|c|c|c|c|c|c|c|c|}
\hline \multirow{2}{*}{ Herbicidas } & \multirow{2}{*}{$\begin{array}{c}\text { Dose i.a. } \\
\left(\mathrm{g} \mathrm{ha}^{-1}\right)\end{array}$} & \multicolumn{2}{|c|}{ Fitointoxicação (\%) } & \multicolumn{2}{|c|}{ Altura $(\mathrm{cm})$} & \multicolumn{2}{|c|}{ Área Foliar $\left(\mathrm{cm}^{2}\right)$} \\
\hline & & $\operatorname{Sim} \underline{1 !}$ & Não & Sim & Não & Sim & Não \\
\hline Chlorimuron-ethyl & 15 & $24 \mathrm{aC}$ & $35 \mathrm{bC}$ & $47,31 \mathrm{aB}$ & $46,35 \mathrm{bB}$ & $1380,00 \mathrm{aB}$ & $1313,80 \mathrm{bB}$ \\
\hline Fluazifop-p-butil & 200 & $0 \mathrm{aA}$ & $0 \mathrm{aA}$ & $48,35 \mathrm{aA}$ & $48,35 \mathrm{aA}$ & $1858,25 \mathrm{aA}$ & $1780,29 \mathrm{bA}$ \\
\hline Fomesafen & 250 & $15 \mathrm{aB}$ & $15 \mathrm{aB}$ & $48,00 \mathrm{aA}$ & $48,18 \mathrm{aA}$ & $1728,25 \mathrm{aAB}$ & $1688,06 \mathrm{bAB}$ \\
\hline [Fluazifop+Fomesafen] ${ }^{2 /}$ & $200+250$ & $25 \mathrm{aC}$ & $40 \mathrm{bC}$ & $48,10 \mathrm{aA}$ & $46,62 \mathrm{bB}$ & $1567,02 \mathrm{aB}$ & $1480,19 b B$ \\
\hline Oxyflurofen & 600 & $33 \mathrm{aC}$ & $40 \mathrm{bC}$ & $47,05 \mathrm{aB}$ & $47,08 \mathrm{aB}$ & $1573,68 \mathrm{aB}$ & $1389,15 b B$ \\
\hline Testemunha & 0 & $0 \mathrm{aA}$ & $0 \mathrm{aA}$ & $48,3 \mathrm{aA}$ & $48,7 \mathrm{aA}$ & $1880,00 \mathrm{aA}$ & $1850,61 \mathrm{aA}$ \\
\hline $\mathrm{CV}(\%)$ & - & \multicolumn{2}{|c|}{12,53} & \multicolumn{2}{|c|}{11,89} & \multicolumn{2}{|c|}{17,98} \\
\hline
\end{tabular}

O chlorimuron-ethyl, oxyfluorfem e [fluazifop-butil+fomesafen] causaram redução da massa seca de raízes tanto das plantas de café inoculadas como nas não inoculadas. Quanto ao efeito da inoculação, verificou-se que as plantas inoculadas foram superiores em relação as não inoculadas na presença e ausência dos herbicidas (Tabela 2).

A massa seca foliar foi influenciada negativamente pela aplicação dos herbicidas chlorimuron-ethyl e oxyfluorfen, tanto para as plantas inoculadas como as não inoculadas. Entretanto, a massa seca das plantas inoculadas foram superiores na aplicação dos herbicidas chlorimuron-ethyl e oxyfluorfen em relação às plantas não inoculadas (Tabela 2).

Analisando o efeito dos herbicidas no volume de raiz de plantas de café, constatou-se que chlorimuron-ethyl, oxyfluorfen e [fluazifop-p-butil+fomesafen] promoveram valores inferiores em relação às plantas sem aplicação, sendo que para as plantas inoculadas, o chlorimuron-ethyl, o oxyfluorfen e o [fluazifop-p-butil+fomosafen] apresentaram menor volume de raízes. Para o efeito de inoculação observou que a presença de todos os herbicidas proporcionou menor incremento no 
volume radicular para as plantas não inoculadas (Tabela 3).

Com plantas cultivadas em vasos em casa de vegetação, Ronchi e Silva (2003) também relataram além da elevada fitotoxicidade $(62,5 \%)$ do oxyfluorfen, redução significativa na altura e biomassa da parte aérea das plantas em 27 e 44\%, respectivamente.

Tabela 2. Massa seca radicular (MSR), foliar (MSF) e volume de raiz (mL) de plantas de café (Catuaí Vermelho IAC 99) inoculadas e não inoculadas com fungos micorrízicos arbusculares (Rhizophagus clarus e Gigaspora margarita), aos 60 DAA.

\begin{tabular}{|c|c|c|c|c|c|c|c|}
\hline \multirow{2}{*}{ Herbicidas } & \multirow{2}{*}{ Dose i.a. $\left(\mathrm{g} \mathrm{ha}^{-1}\right)$} & \multicolumn{2}{|c|}{$\operatorname{MSR}(\mathrm{g})$} & \multicolumn{2}{|c|}{$\operatorname{MSF}(\mathrm{g})$} & \multicolumn{2}{|c|}{ Volume de Raiz (mL) } \\
\hline & & $\operatorname{Sim}^{1 /}$ & Não & $\operatorname{Sim}^{\underline{1}}$ & Não & $\operatorname{Sim}^{1 /}$ & Não \\
\hline Chlorimuron-ethyl & 15 & $14,00 \mathrm{aB}$ & $11,25 \mathrm{bB}$ & $19,75 \mathrm{aB}$ & $17,25 \mathrm{bB}$ & $45,00 \mathrm{aC}$ & $41,00 \mathrm{bB}$ \\
\hline Fluazifop-p-butil & 200 & $15,00 \mathrm{aA}$ & $13,00 \mathrm{bA}$ & $22,15 \mathrm{aA}$ & $22,89 \mathrm{aA}$ & $49,25 \mathrm{aA}$ & $47,00 \mathrm{bA}$ \\
\hline Fomesafen & 250 & $15,00 \mathrm{aA}$ & $13,25 \mathrm{bA}$ & $21,50 \mathrm{aA}$ & $21,75 \mathrm{aA}$ & $49,05 \mathrm{aA}$ & $47,50 \mathrm{bA}$ \\
\hline [Fluazifop+Fomesafen] ${ }^{2 l}$ & $200+250$ & $14,00 \mathrm{aB}$ & $12,25 \mathrm{bAB}$ & $20,75 \mathrm{aAB}$ & $20,40 \mathrm{aAB}$ & $47,00 \mathrm{aB}$ & $42,25 \mathrm{bB}$ \\
\hline Oxyfluorfen & 600 & $13,00 \mathrm{aB}$ & $10,95 \mathrm{bB}$ & $18,50 \mathrm{aB}$ & $15,72 \mathrm{bB}$ & $44,50 \mathrm{aC}$ & $40,42 \mathrm{bB}$ \\
\hline Testemunha & 0 & $15,75 \mathrm{aA}$ & $13,00 \mathrm{bA}$ & $22,00 \mathrm{aA}$ & $23,00 \mathrm{aA}$ & $50,00 \mathrm{aA}$ & $47,50 \mathrm{aA}$ \\
\hline $\mathrm{CV}(\%)$ & - & & ,91 & & 9 & & \\
\hline
\end{tabular}

Tabela 3. Teores fósforo (P) e ferro (Fe) de plantas de café (Catuaí Vermelho IAC 99) inoculadas e não inoculadas com fungos micorrízicos arbusculares (Rhizophagus clarus e Gigaspora margarita), aos 60 DAA.

\begin{tabular}{|c|c|c|c|c|c|}
\hline \multirow{2}{*}{ Herbicidas } & \multirow{2}{*}{$\begin{array}{c}\text { Dose i.a. } \\
\left(\mathrm{g} \mathrm{ha}^{-1}\right)\end{array}$} & \multicolumn{2}{|c|}{$\mathrm{P}(\mathrm{g} / \mathrm{kg})$} & \multicolumn{2}{|c|}{$\mathrm{Fe}(\mathrm{mg} / \mathrm{kg})$} \\
\hline & & $\operatorname{Sim} 1 /$ & Não & $\operatorname{Sim} 1 /$ & Não \\
\hline Clorimurom & 15 & $1,09 \mathrm{aB}$ & $1,01 \mathrm{bB}$ & $192,90 \mathrm{aB}$ & $190,56 \mathrm{aB}$ \\
\hline Fluazifop & 200 & $1,18 \mathrm{aA}$ & $1,12 \mathrm{bA}$ & $212,56 \mathrm{aA}$ & $210,65 a A$ \\
\hline Fomesafen & 250 & $1,15 \mathrm{aA}$ & $1,09 \mathrm{bA}$ & $213,70 \mathrm{aA}$ & $209,80 \mathrm{bA}$ \\
\hline [Fluazifop+Fomesafen] ${ }^{2 /}$ & $200+250$ & $1,07 \mathrm{aB}$ & $0,98 \mathrm{bB}$ & $198,80 \mathrm{aB}$ & $180,90 b C$ \\
\hline Oxyfluorfen & 600 & $1,01 \mathrm{aB}$ & $0,85 \mathrm{bC}$ & $190,76 \mathrm{aB}$ & $174,04 b C$ \\
\hline Testemunha & 0 & $1,25 \mathrm{aA}$ & $1,10 \mathrm{bA}$ & $215,90 \mathrm{aA}$ & $211,98 \mathrm{bA}$ \\
\hline $\mathrm{CV}(\%)$ & - & \multicolumn{2}{|c|}{7,09} & \multicolumn{2}{|c|}{12,71} \\
\hline
\end{tabular}

Em relação aos teores de nutrientes, houve efeitos significativos $(\mathrm{p}<0,05)$ para o fósforo $(\mathrm{P})$, ferro $(\mathrm{Fe})$, potássio $(\mathrm{K})$ e zinco $(\mathrm{Zn})$. Os teores desses nutrientes foram afetados pela aplicação de chlorimuron-ethyl, oxyfluorfen e [fluazifop-p-butil+fomesafen]. Os teores de P para as plantas inoculadas foram superiores em todos os tratamentos em relação as não inoculadas. Os teores de $\mathrm{Fe}$ apenas com aplicação de chlorimuron-ethyl e fluazifop-pbutil não diferiram em relação as plantas inoculadas e não inoculadas. Quanto aos teores de $\mathrm{K}$, as plantas submetidas a aplicação de chlorimuron-ethyl e oxyfluorfen se sobressaíram quando houve a inoculação com FMAs. Para os teores de Zn, apenas a testemunha se diferenciou entre inoculada e não inoculada, a planta inoculada obteve maior teor que plantas não inoculadas (Tabelas 3 e 4).

Os menores teores dos nutrientes com a aplicação de chlorimuron-ethyl, oxyfluorfen e fluazifop-p-butil+fomesafen pode ser explicado pelos danos causados na estrutura das plantas, diminuindo assim sua capacidade de absorção de nutriente por apresentar menor sistema radicular, juntamente com a redução de sua capacidade fotossintética. De acordo com Ronchi e Silva (2003; 2004) e Magalhães et al. (2010), os sintomas visuais do oxyfluorfen e [fluazifop-butil+fomesafen] em café reduziram a área fotossintética das plantas. 
Analisando a colonização do sistema radicular por FMAs, notou-se que, com a presença dos herbicidas fomesafen, chlorimuron-ethyl, oxyfluorfen e [fluazifop-pbutil+fomesafen], as plantas foram afetadas independente da inoculação. As plantas inoculadas com o mix de fungos selecionados obtiveram maior colonização das raízes, do que as plantas não inoculadas que foram colonizadas apenas por FMAs nativos do solo utilizado
(Tabela 5). Na literatura, alguns trabalhos evidenciam o efeito de herbicidas em préemergência, como o sulfentrazone, sobre microorganismos como a menor colonização de FMAs em soja (Vieira et al., 2007). Para o feijoeiro, Santos et al. (2006) observaram que a colonização de FMAs foi reduzida em até 12 dias após aplicação em pós-emergência dos herbicidas fomesafen e fluazifop-p-butil.

Tabela 4. Teores potássio (K) e zinco ( $\mathrm{Zn}$ ) de plantas de café (Catuaí Vermelho IAC 99) inoculadas e não inoculadas com fungos micorrízicos arbusculares (Rhizophagus clarus e Gigaspora margarita), aos 60 DAA.

\begin{tabular}{|c|c|c|c|c|c|}
\hline \multirow{2}{*}{ Herbicidas } & \multirow{2}{*}{ Dose i.a. $\left(\mathrm{g} \mathrm{ha}^{-1}\right)$} & \multicolumn{2}{|c|}{$\mathrm{K}(\mathrm{g} / \mathrm{kg})$} & \multicolumn{2}{|c|}{$\mathrm{Zn}(\mathrm{mg} / \mathrm{kg})$} \\
\hline & & $\operatorname{Sim}^{1 /}$ & Não & $\operatorname{Sim} \underline{1}$ & Não \\
\hline Chlorimuron-ethyl & 15 & $17,90 \mathrm{aB}$ & $13,41 \mathrm{bB}$ & $3,61 \mathrm{aB}$ & $3,41 \mathrm{aB}$ \\
\hline Fluazifop-p-butil & 200 & $18,98 \mathrm{aA}$ & $18,90 \mathrm{aA}$ & $4,05 \mathrm{aA}$ & $4,01 \mathrm{aA}$ \\
\hline Fomesafen & 250 & $18,90 \mathrm{aA}$ & $18,39 \mathrm{aA}$ & $4,01 \mathrm{aA}$ & $3,90 \mathrm{aA}$ \\
\hline [Fluazifop+Fomesafen] ${ }^{2 /}$ & $200+250$ & $15,52 \mathrm{aC}$ & $15,10 \mathrm{aB}$ & $3,12 \mathrm{aC}$ & $3,18 \mathrm{aB}$ \\
\hline Oxyfluorfen & 600 & $14,56 \mathrm{aC}$ & $12,34 \mathrm{bB}$ & $3,01 \mathrm{aC}$ & $3,10 \mathrm{aB}$ \\
\hline Testemunha & 0 & $19,45 \mathrm{aA}$ & $18,92 \mathrm{aA}$ & $4,15 \mathrm{aA}$ & $4,09 \mathrm{bA}$ \\
\hline $\mathrm{CV}(\%)$ & - & \multicolumn{2}{|c|}{10,01} & \multicolumn{2}{|c|}{12,98} \\
\hline
\end{tabular}

Tabela 5. Colonização micorrízica de plantas de café (Catuaí Vermelho IAC 99) inoculadas e não inoculadas com fungos micorrízicos arbusculares (Rhizophagus clarus e Gigaspora margarita), aos 60 DAA.

\begin{tabular}{|c|c|c|c|}
\hline \multirow{2}{*}{ Herbicidas } & \multirow{2}{*}{$\begin{array}{c}\text { Dose i. a. } \\
\left(\mathrm{g} \mathrm{ha}^{-1}\right)\end{array}$} & \multicolumn{2}{|c|}{ Colonização (\%) } \\
\hline & & $\operatorname{Sim} \underline{1 /}$ & Não \\
\hline Chlorimuron-ethyl & 15 & $60,21 \mathrm{aC}$ & $42,47 \mathrm{bB}$ \\
\hline Fluazifop-p-butil & 200 & $70,33 \mathrm{aAB}$ & $45,33 \mathrm{bA}$ \\
\hline Fomesafen & 250 & $65,62 \mathrm{aB}$ & $43,13 \mathrm{bAB}$ \\
\hline [Fluazifop+Fomesafen] ${ }^{2 /}$ & $200+250$ & $59,44 \mathrm{aC}$ & $41,31 \mathrm{bB}$ \\
\hline Oxyfluorfen & 600 & $59,65 \mathrm{aC}$ & $39,91 \mathrm{bB}$ \\
\hline Testemunha & 0 & $72,3 \mathrm{aA}$ & $45,95 \mathrm{bA}$ \\
\hline $\mathrm{CV}(\%)$ & - & & \\
\hline
\end{tabular}

\section{Conclusões}

O fluazifop-p-butil apresentou-se seletivo para as plantas de café arábica cultivar Catuaí Vermelho IAC 99, não causando danos a colonização dos fungos micorrízicos arbusculares. Dessa forma, mostra-se mais promissor para aplicação em área total em plantas de café.
No geral, as plantas de café inoculadas com FMAs foram mais tolerantes aos efeitos negativos dos herbicidas chlorimuron-ethyl, fomesafen, oxyfluorfen e [fluazifop-pbutil+fomesafen].

\section{Agradecimentos}

À CAPES, FAPEMIG, CNPq e FAPESC pela concessão dos recursos e bolsas 
para a realização deste trabalho.

\section{Referências}

ALCÂNTARA, E.N. et al. Avaliação de herbicidas para cafeeiros em formação. In: SIMPÓSIO DE PESQUISA DOS CAFÉS DO BRASIL, 1., 2000, Poços de Caldas, MG. Resumos Expandidos... Brasília, DF: EMBRAPA CAFÉ, 2000. p.967-970.

\section{ASSOCIATION OF OFFICIAL} ANALYTICAL CHEMISTS - AOAC. Official methods of analysis. 12.ed. Washington DC: 1975. 1094p.

BRAGA, J.M.; FELLIPO, B.V. Determinação espectrofotométrica de $\mathrm{P}$ em extratos de solo e material vegetal. Revista Ceres, v.21, n.113, p.73-85, 1974.

CAIXETA, G.Z.T. et al. Gerenciamento como forma de garantir a competitividade da cafeicultura. Informe Agropecuário, v.29, n.247, p.14-23, 2008.

CARRIGAN, L.; FREY, K.J. Root volumes of Avena species. Crop Science, v.20, n.3, p.407408, 1980.

COLOZZI FILHO, A. et al. Efetividade de diferentes fungos micorrízicos arbusculares na formação de mudas, crescimento pós transplante e produção do cafeeiro. Pesquisa Agropecuária Brasileira, Brasília, v.29, n.9, p.1397-1406, 1994.

COLOZZI FILHO, A.; CARDOSO, E.J.B.N. Detecção de fungos micorrízicos arbusculares em raízes de cafeeiro e de crotalária cultivada na entrelinha. Pesquisa Agropecuária Brasileira, v.35, n.10, p.2033-2042, 2000.

CONAB, Companhia Nacional de Abastecimento. Acompanhamento da Safra Brasileira Café. Segunda estimativa safra maio/2013. Brasilia: CONAB 2012. Disponivel em

http://www.conab.gov.br/OlalaCMS/uploads/ar quivos/13_05_14_09_35_12_boletim_cafe_ma io_2013.pdf, acesso em 28 de junho de 2013.
GIOVANNETTI, J.W.; MOSSE, B. An evaluation of techniques for measuring vesicular arbuscular mycorrhizal infection in roots. New Phytology, v.48, n.3, p.489-500, 1980

GUIMARÃES, P.T.G. et al. Cafeeiro. In: RIBEIRO, A.C.; GUIMARÃES, P.T.G.; ALVAREZ V.V.H. (Eds.). Recomendações para uso de corretivos e fertilizantes em Minas Gerais: $5^{\mathbf{a}}$ aproximação. Viçosa, MG: Comissão de Fertilidade do Solo do Estado de Minas Gerais, 1999. p.289-302.

JACKSON, M.L. Soil chemical analysis. Englewood Cliffs: Prentice Hall, 1958. 458p.

KJELDAHL, J.A new method for the determination of nitrogen in organic matter. Zeitschreft fur Analytische Chemie, v.22, n.1, p.366 1883 .

LOCATELLI, L.M.; LOVATO, P.E. Inoculação micorrízica e aclimatização de dois porta-enxertos micropropagados de macieira. Pesquisa Agropecuária Brasileira v.37, n.2, p.177-184, 2002.

MAGALHÃES, C.E.O. et al. Seletividade e controle de plantas daninhas com oxyfluorfen e sulfentrazone na implantação de lavoura de café. Planta Daninha, v.30, n.3, p.607-616, 2012.

MIRANDA, G.R.B. et al. Formação de mudas de cafeeiro em substratos oriundos de diferentes métodos de desinfestação. Bragantia, v.65, n.2, p.303-307. 2006.

MOREIRA, F.M.S.; SIQUEIRA, J.O. Microbiologia e Bioquímica do Solo. $2^{\mathrm{a}}$ ed. atual. e ampl. Lavras: Editora UFLA, 2006. $729 \mathrm{p}$.

POUYU-ROJAS, E.; SIQUEIRA, J.O. Micorriza arbuscular e fertilização do solo no desenvolvimento pós-transplante de mudas de sete espécies florestais. Pesquisa Agropecuária Brasileira, v.35, n.1, p.103-114, 2000. 
RONCHI, C.P.; SILVA, A.A. Tolerância de mudas de café a herbicidas aplicados em pósemergência. Planta Daninha, v.21, n.3, p.421426, 2003.

RONCHI, C.P.; SILVA, A.A. Weed control in young coffee plantations through postemergence herbicide application onto total area. Planta Daninha, v.22, n.4, p.607-615, 2004.

RONCHI, C.P.; SILVA, A.A.; FERREIRA, L.R. Manejo de plantas daninhas em lavouras de café. Viçosa, MG: Universidade Federal de Viçosa, Departamento de Fitopatologia, 2001, 94p.

RONCHI, C.P.; TERRA, A.A.; SILVA, A.A. Growth and nutrient concentration in coffee root system under weed species competition. Planta Daninha, v.25, n.4, p.679-687, 2007.

SILVA, A.A.; FERREIRA, F.A.; FERREIRA, L.R. Herbicidas: Classificação e Mecanismos de Ação. In: SILVA, A. A.; SILVA, J. F. Tópicos em manejo de plantas daninhas. Viçosa, MG: Universidade Federal de Viçosa, 2009. p.83148.

SILVA, A.A.; RONCHI, C.P. Avanços nas pesquisas sobre o controle de plantas daninhas na cultura do café. In: ZAMBOLIM, L. (Ed.). Produção integrada de café. Viçosa, MG: Universidade Federal de Viçosa, 2003. p.103132.

SILVA, D.V. et al. Seletividade de herbicidas pós-emergentes na cultura da mandioca. Planta Daninha, v.30, n.4, p.835-841, 2012.

VIEIRA, R.F.; SILVA, C.M.M.S.; SILVEIRA, A.P.D. Soil microbial biomass $\mathrm{C}$ and symbiotic processes associated with soybean after sulfentrazone herbicide application. Plant Soil, v.300, n.1, p.95-103, 2007. 